\title{
Procedural and follow-up insights from optical coherence tomography-guided management of intracoronary stent loss and crushing
}

\author{
Lorenzo Azzalini, Satoru Mitomo, Luciano Candilio, Antonio Colombo \\ Division of Interventional Cardiology, Cardio-Thoracic-Vascular Department, \\ San Raffaele Scientific Institute, Milan, Italy
}

A 55-year-old man underwent left anterior descending (LAD) chronic total occlusion intervention (Fig. 1A, B).

The occlusion was crossed antegradely and predilated (Fig. 1C). During delivery of a $3.0 \times$ $\times 33-\mathrm{mm}$ stent, the operator encountered significant resistance, and soon noticed stent dislodgment from the delivery system. Since the stent appeared deformed and was located in front of the circumflex and first diagonal (D1) ostia (Fig. 1D), the operator chose to crush it (Fig. 1E). A $3.5 \times$ $\times 33$-mm stent (Fig. $1 \mathrm{~F}$ ) was then implanted and post-dilated with a $4.5-\mathrm{mm}$ non-compliant balloon. Optical coherence tomography revealed that stent crushing also involved a $3.5 \times 9 \mathrm{~mm}$ (likely malapposed) stent that was implanted across the LAD-D1 bifurcation 2 months earlier, which might have caused subsequent stent dislodgment in the following intervention. Final result was good (Fig. $1 \mathrm{G}, \mathrm{H})$. Optical coherence tomography confirmed optimal stent crushing (Fig. 1a-d). The patient was discharged on long-term dual antiplatelet therapy (aspirin + ticagrelor).

A 5-month follow-up coronary angiogram was performed, which confirmed stent patency. Optical coherence tomography showed complete endothelialization of the crushed stents with embedment into the vessel wall (Fig. 1a'-d', Supplementary Video 1 - see journal website). Therefore, the decision was made to discontinue ticagrelor after completing a 12-month course.

Stent loss is a rare but serious percutaneous coronary intervention complication. Management strategies include implantation, retrieval, or crushing. Crushing is preferred when the dislodged stent is deformed and/or in an inappropriate location, like in this case. An intravascular imaging-based management is essential, first to demonstrate adequate crushing, and subsequently to confirm full endothelialization of the crushed stent on follow-up, thus reassessing the risk of stent thrombosis and possibly avoiding prolonged dual antiplatelet therapy.

Conflict of interest: Dr. Azzalini reports having received research support from ACIST Medical Systems and honoraria from Guerbet. The other authors have no disclosures to report.

Address for correspondence: Lorenzo Azzalini, MD, PhD, MSc, Division of Interventional Cardiology, Cardio-Thoracic-Vascular Department, San Raffaele Scientific Institute, Via Olgettina 60, 20132 Milan, Italy, tel: +390226437331, fax: +390226437339, e-mail: azzalini.lorenzo@hsr.it 


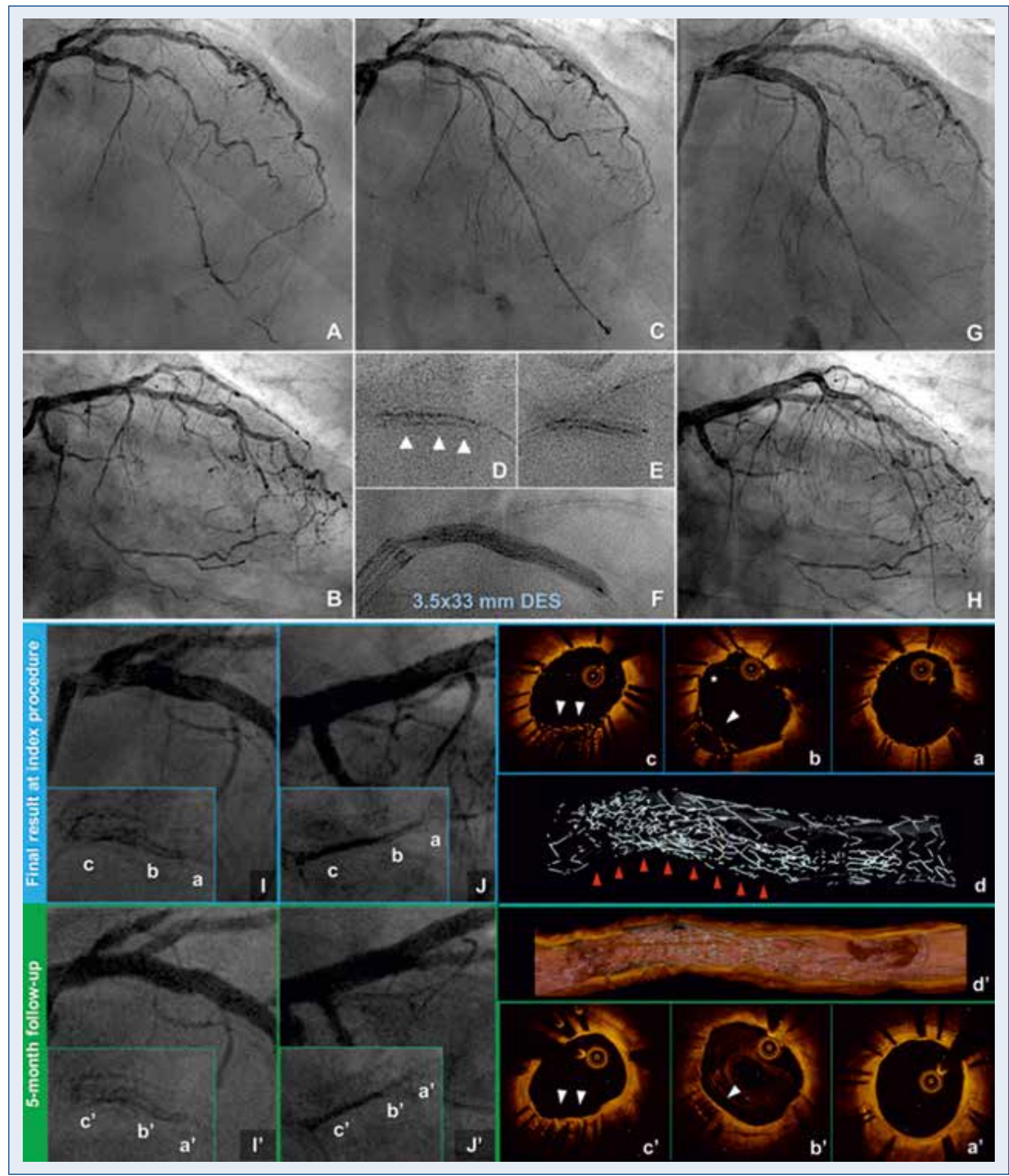

Figure 1. A, B. Mid left anterior descending chronic total occlusion. The occlusion was crossed with antegrade wire escalation and predilated (C). D. A $3.0 \times 33-\mathrm{mm}$ drug-eluting stent (DES) was dislodged from the delivery system, appeared severely distorted (arrowheads) and was located in front of the circumflex and first diagonal ostia. E. Balloon crushing of the dislodged stent was performed. F. A $3.5 \times 33-\mathrm{mm}$ DES was implanted and post-dilated, G, H. with optimal final result. In the lower panel, angiographic and optical coherence tomography (OCT) images of the final result after the index procedure in (I) cranial and ( $\mathbf{J}$ ) caudal projections are shown in detail. a-c. Optimal stent apposition is observed. Arrowheads in (b, $\mathbf{c}$ and $\mathbf{d}$ ) point at the crushed stents. The asterisk in panel $\mathbf{b}$ indicates that just one layer of metal is seen on the opposite side with regard to the crushed segment, thus suggesting that crushing also involved a short (likely malapposed) stent that had been previously implanted across the left anterior descending-first diagonal bifurcation. At 5-month follow-up coronary angiogram ( $\mathbf{I}^{\prime}$ and $\mathbf{J}^{\prime}$ ), a good result is observed and confirmed by OCT imaging $\left(\mathbf{a}^{\prime}-\mathbf{d}^{\prime}\right)$. Arrowheads in panel $\mathbf{b}^{\prime}$ and $\mathbf{c}^{\prime}$ point at the crushed stents, which are now fully endothelialised. 